\title{
Coulisses
}

Revue de théâtre

\section{Bertold Brecht, La Bonne âme de Setchouan}

Mise en scène : Anne-Margrit Leclerc

\section{David Ball}

\section{(2) OpenEdition}

\section{Journals}

Édition électronique

URL : https://journals.openedition.org/coulisses/819

DOI : 10.4000/coulisses.819

ISSN : 2546-9460

Éditeur

Presses universitaires de Franche-Comté

\section{Édition imprimée}

Date de publication : 31 décembre 2010

Pagination : 23-26

ISBN : 978-2-84867-302-8

ISSN : $1150-594 \mathrm{X}$

Référence électronique

David Ball, « Bertold Brecht, La Bonne âme de Setchouan », Coulisses [En ligne], 41 | Automne 2010, mis en ligne le 30 novembre 2016, consulté le 29 décembre 2022. URL : http://journals.openedition.org/ coulisses/819; DOI : https://doi.org/10.4000/coulisses.819 


\section{Bertold Brecht, La Bonne âme de Setchouan}

Mise en scène : Anne-Margrit Leclerc

David Ball

\section{RÉFÉRENCE}

Bertold Brecht, La Bonne âme de Setchouan, mise en scène : Anne-Margrit Leclerc, représentation au théâtre de l'Allan, Montbéliard, novembre 2009

1 Avec ses collaboratrices, Brecht consacra de longues années d'exil à l'élaboration de cette parabole pour le théâtre. Pourrait-on aller jusqu'à dire que sa vraie bonne âme est Margarethe Steffin, la collaboratrice avec qui il travailla chaque réplique, mot par mot? Ou peut-être, avant elle, Elisabeth Hauptmann? Ce fut grâce à elle qu'il prit connaissance du théâtre oriental, en lisant ses traductions des pièces nô japonaises à partir de l'anglais d'Arthur Waley. Le souci principal de Brecht pendant ce long processus d'écriture était de donner à une séquence de contrastes et de paradoxes qui risquait d'être trop schématique l'effet du réel, le corsé du vécu. Et pourtant, c'est justement dans sa clarté schématique que la fable trouve sa force, une clarté qui se prête néanmoins à des interprétations différentes. Trop de détails risquent d'alourdir la démonstration, d'autant plus que c'est plutôt dans les détails que le penchant pédagogique de Brecht se manifeste : le désir de tout expliquer.

Rappelons les lignes principales de l'histoire. Les dieux descendent sur terre pour voir s'il est possible d'y vivre tout en obéissant à leurs commandements. Ils constatent qu'en effet ce n'est pas facile. Mais ils dénichent enfin une assez bonne âme en la personne de Chen Té, une prostituée au cœur d'or, et en partant, ils lui donnent mille dollars d'argent, avec lesquels elle achète un débit de tabac. Mais une telle bande de bras cassés se met à profiter de sa générosité qu'elle est obligée d'inventer et d'assumer une deuxième identité, celle de son cousin, Chui Ta, homme sans pitié qui se débarrasse des bouches inutiles et fait fructifier l'affaire au point que le débit se transforme en usine de tabac. La question posée est claire, bien avant l'épilogue : comment agir avec justice 
dans un monde injuste? A nous d'y répondre. Mais impossible de ne pas voir que l'auteur nous a déjà fourni la réponse nécessaire : que c'est le monde qu'il faut changer.

Dès le discours initial de Wang, le vendeur d'eau, nous nous rendons compte de tout ce que Brecht a emprunté au théâtre oriental. Peut-on imaginer Ibsen commencer une pièce par: Nora, se présentant au public: Je suis Nora, la poupée. Je suis très heureuse : mon mari, Torvald, a un bon poste à la banque, j'ai deux enfants adorables et nous habitons un bel appartement. Si je suis moi-même ici devant la porte, c'est parce que j'attends d'un moment à l'autre l'arrivée de mon amie, Christine, que je n'ai pas vue depuis longtemps, et à qui j'ai des révélations à faire qui vont l'étonner...

Les autres partis pris de Brecht dans cette pièce sont, par contre, plutôt conservateurs. Il semble avoir fait sienne l'idée des réformateurs du dix-neuvième siècle que la seule cause du crime est la pauvreté et qu'avec son abolition il disparaîtrait. Nous ne savons que trop bien aujourd'hui qu'il n'en est rien. Et sa conception du caractère individuel semble ignorer ou s'opposer aux découvertes de la psychanalyse. Le caractère de Chen Té est présenté si entier, si stable, qu'elle a besoin de s'en construire un autre pour s'adapter aux circonstances. Même sa profession de prostituée semble plutôt l'effet de sa générosité.

5 Mais «sembler» est sans doute ici le mot juste, car les positions de Brecht sont rarement aussi simples que sa réputation de pédagogue et de propagandiste laisse à penser. Le comportement des personnages est compliqué par au moins deux facteurs : la faiblesse et l'amour. La faiblesse physique et morale des gens qui profitent de la générosité de Chen Té suggère que le mal n'est pas simplement une question de moyens financiers. Et puis l'amour! Comment régler et intégrer l'amour dans la société parfaite? Chen Té est sans illusion sur la sincérité de son amoureux, Yang Soun. Elle sait que pour lui l'ambition de devenir pilote d'avion est plus importante que son affection pour elle et que pour réaliser son ambition il est prêt à se servir de n'importe qui. A la fin de la cinquième scène, elle le choisit pourtant: «Je veux aller avec celui que j'aime. »

6 Quant à la psychanalyse, une interprétation freudienne, et même lacanienne, du personnage principal n'est pas du tout à exclure. Que, par exemple, être femme est un état provisoire, instable, divisé entre le désir d'assumer une masculinité dominante et valorisante et le recours à une féminité qui tente de faire sa force d'une faiblesse séductrice. Une telle interprétation est facilitée non seulement par les hésitations et les contradictions de Chen Té à l'égard de Yang Soun, mais aussi, dans cette mise en scène, par le fait que l'actrice qui joue le rôle de Chen Té ne met pas de masque pour jouer le rôle de Chui Ta mais change simplement de costume. Le masque, prévu par Brecht, indiquerait que le caractère de Chen Té reste inchangé en dessous, que l'identité de Chui Ta ne vient pas d'elle mais de l'extérieur. Plus généralement, le masque du théâtre oriental, qui permet d'abord aux hommes de jouer les rôles de femme et qui fait ensuite office de maquillage voyant et frappant, suggère que le caractère est fixe.

Dans son récit, L'Étrange Cas du Docteur Jekyll et de Mister Hyde, Stevenson présente le phénomène de la double personnalité comme problème purement psychologique. C'est du tréfonds du caractère du docteur lui-même que surgit l'identité mauvaise de Mister Hyde, identité qui devient de plus en plus dominante, jusqu'au point où il ne peut s'en débarrasser qu'en se suicidant. C'est grâce à une découverte scientifique mystérieuse que les transformations sont effectuées, découverte qui relève pourtant davantage de la magie noire que de la pure science. Brecht lui aussi avait peur des possibilités de la 
science, une peur plus mesurée et rationnelle. Suivant la destruction des villes japonaises par la bombe atomique à la fin de la deuxième guerre, il modifia sa pièce, $L a$ Vie de Galilée, pour mettre l'accent encore plus clairement sur la responsabilité morale et sociale du scientifique.

Dans la mise en scène de La Bonne Âme d'Anne-Margrit Leclerc, huit acteurs assurent la totalité des rôles avec panache et fluidité : Sylvie Amato, Stéphanie Farison, Nadine Ledru, Laëtitia Pitz, Laurent Fraunié, Hervé Lang, Valéry Plancke et Yves Thouvenel. La toile de fond du décor a un air de peinture orientale : des rizières d'un vert terne sous un grand ciel gris. Trois échafaudages mobiles et deux rideaux, du rouge vif cher aux Chinois, permettent aux acteurs de composer les décors ponctuels du débit de tabac, de la rue, du parc municipal, de la place publique, du restaurant et de l'usine. (Scénographie : Grégoire Faucheux.) La scène du procès à la fin se joue devant le grand rideau du théâtre. Ce qui surprend un peu, car cette scène nous semble plutôt celle de l'ouverture et de la révélation, mais le grand rideau sombre suggère peut-être un effet d'huis clos. Les costumes sont modernes, des années d'entre-deux-guerres. Les dieux portent des châles ou des manteaux. Le troisième dieu, joué par une actrice, a une allure de sorcière. Les deux autres portent des lunettes, suggérant une myopie plus intellectuelle que visuelle. (Costumes : Alexandra Wassef.)

9 Le jeu des acteurs a toute la rapidité préconisée par l'auteur, et son texte a été à cet effet un peu élagué. Mais Brecht avait l'habitude de réviser ses pièces selon les besoins des compagnies et des théâtres où elles étaient produites. En 1943 il fit de La Bonne Âme la version dite américaine, où les transactions autour des 500 dollars dont Yang Soun a besoin pour se faire embaucher comme pilote sont simplifiées mais où en revanche le commerce de tabac est changé en trafic d'opium, ce qui complique et noircit considérablement le dilemme moral du personnage principal.

10 Pour la mise en scène de Montbéliard, plusieurs chansons ont été, heureusement, réduites ou éliminées. Les chansons de Brecht, censées être légères et populaires, sont probablement aujourd'hui l'élément le plus difficile et dissuasif de son théâtre (de son théâtre non musical). Et en traduction, évidemment, elles sont encore plus difficiles à faire passer.

11 Et la distanciation dans tout cela? Le fameux Verfremdungseffekt que Brecht théorisa après sa rencontre à Moscou en 1935 avec l'acteur chinois, Mei Lan-fang, qui lui fit découvrir un style de jeu où l'acteur ne se perdait pas dans son rôle mais restait toujours visiblement acteur conscient d'un public en train de le regarder. Question difficile. Et la sincérité des acteurs, question analogue : est-ce celle des artisans qui s'efforcent de faire un travail honnête ou des adeptes de la méthode stanislavskienne qui font leurs les émotions des personnages? Les deux ne sont pas forcément incompatibles. Disons dans ce cas que le jeu est tout ce qu'il nous faut pour comprendre et apprécier l'histoire, avec toutefois une force et une énergie dans les répliques et les gestes qui font penser plutôt à la sincérité de l'identification.

12 Les acteurs s'adressent au public et se servent de tout l'espace du théâtre, dont l'orchestre et le premier balcon. L'Allan de Montbéliard est un théâtre traditionnel à coupole, aux proportions harmonieuses. Et le demi-cercle profond de ses balcons semble exprimer un désir d'échange citoyen qui convient parfaitement au théâtre de Brecht.

Dans son désir de créer un théâtre épique d'idées et de contestation, il ne fut pas le seul de son époque à être influencé par le théâtre oriental. Paul Claudel, lui aussi influencé 
encore plus directement par les pièces nô qu'il avait eu l'occasion de voir au Japon, fut peut-être à son tour une influence. Brecht admirait son écriture théâtrale tout en déplorant ses idées politiques réactionnaires. Il connaissait sans doute sa pièce, Christophe Colomb, avec musique composée par Darius Milhaud, qui fut jouée à Berlin en 1930. John Willett, dans son livre de 1977 sur le théâtre de Brecht, compare de manière plutôt amusante les deux écrivains :

Ils furent influencés tous deux par Rimbaud, la Bible et le théâtre du nô; ils se servirent tous deux du théâtre à des fins didactiques; ils situèrent tous deux l'action de leurs pièces dans des lieux lointains ou fantasques; ils se plurent tous deux à briser l'illusion théâtrale mais eurent des difficultés à donner l'illusion de la vie à leurs personnages. Ils collaborèrent avec des compositeurs qui étaient aussi des amis personnels. Et ils divergèrent tous deux de leurs traditions nationales: Claudel parce qu'il se servit du français pour exprimer des idées vagues et Brecht parce qu'il se servit de l'allemand pour exprimer des idées précises.

INDEX

oeuvrecitee Bonne âme de Setchouan (La)

Palavras-chave : cenografia

Palabras claves : puesta en escena

Keywords : mise-en-scène

Mots-clés : mise en scène 